\title{
Síndrome de Burnout en el personal de enfermería y su relación con cuatro variables laborales
}

\section{Burnout syndrome among nursing staff and its association with four work-related variables}

\section{Síndrome de Burnout no pessoal de enfermagem e sua relação com quatro variáveis laborais}

\author{
E. Rivas ${ }^{a}$, A. Barraza-Macías ${ }^{b, *}$ \\ ${ }^{a}$ Unidad de Terapia Intensiva Adultos, Hospital Materno Infantil de la Ciudad de Durango, Durango, México. \\ b Área de Investigación y Posgrado de la Universidad Pedagógica de Durango, Ciudad de Durango, Durango, \\ México.
}

Recibido: 19 abril 2017

Aceptado: 17 marzo 2018

\begin{abstract}
Resumen
Objetivo: Determinar si las variables: turno de trabajo, situación laboral, el realizar horas laborales extras y servicio en el que se encuentra actualmente, establecen diferencias en el nivel con que se presenta el síndrome de Burnout en los profesionales de enfermería del Hospital Materno Infantil de la ciudad de Durango.

Método: Se realizó un estudio correlacional, transversal y no experimental a partir de la aplicación del Maslach Burnout Inventory-General Survey, a 204 enfermeras/os del Hospital Materno Infantil de la ciudad de Durango, en México.

Resultados: De las cuatro variables estudiadas (turno de trabajo, situación laboral, el realizar horas laborales extras y servicio en el que se encuentra actualmente), es la del turno laboral la que marca una diferencia significativa en el nivel en que se presenta el síndrome de Burnout en el personal de enfermería. Conclusiones: Las condiciones laborales pueden desencadenar situaciones estresantes que a la larga generen el síndrome de Burnout, tal es el caso de variables como turno en que se labora o el tipo de nombramiento laboral que se posea. La aparición de este síndrome afecta el desempeño profesional del personal de enfermería.
\end{abstract}

Palabras clave: Burnout; agotamiento profesional; estrés laboral; despersonalización; enfermería; México.

*Autor para correspondencia. Correo electrónico: tbarraza-2017@hotmail.com (A. Barraza-Macías) 


\begin{abstract}
Objective: To determine if the variables Work Shift, Labor Situation, Extra Hours, and Service Area, make differences in the levels of Burnout among nursing personnel working in the Mother and Child Hospital of the city of Durango, Mexico. Method: This is a correlational, transversal, and not-experimental study which uses the Maslach Burnout Inventory-General Survey to estimate the level of Burnout of 204 nurses working in the Mother and Child Hospital of the City of Durango, Mexico.

Results: From the four studied variables, Work Shift induced significant differences on the levels of reported Burnout.

Conclusions: Conditions such as Shift and Position can trigger stressful situations which in the long term can make the nursing personnel develop the Burnout syndrome which profoundly affects their professional performance.
\end{abstract}

Keywords: Burnout, professional; occupational stress; depersonalization; nursing; Mexico.

\title{
Resumo
}

Objetivo: Determinar se as variáveis mudam, status de emprego, horas de trabalho e realizar serviço extra que está atualmente, estabelecer diferenças na prevalência da síndrome de Burnout em enfermeiros Hospital Materno-Infantil na cidade de Durango.

Método: Um estudo transversal de correlação, não experimental foi conducido a partir da aplicação do Inventário-geral Pesquisa Maslach Burnout 204 enfermeiras/os Hospital Materna e infantil na cidade de Durango, México.

Resultados: De las quatro variáveis estudadas (turno de trabajo, situacão laboral, realização de exercícios laborais extras e serviço em que se encontra ativo), é o mercado de trabalho que marca a diferença significativa no nível com que se apresenta o síndrome de Burnout en personal of enfermería.

Conclusões: As condições laborales podem gerar situações estresantes que a larga generada o síndrome de Burnout, tal é o caso de variáveis como virar em que se labora ou o tipo de nomeação laboral que se posea. A aparição deste síndrome afecta o desempenho profissional do pessoal da enfermagem.

Palavras chave: Burnout; esgotamento profissional; estresse ocupacional; despersonalização; enfermagem; México.

\section{Introducción}

El ambiente laboral en el que se desenvuelve el personal de enfermería suele generar un alto nivel de estrés; el exceso de carga de trabajo, turnos laborales extras, el hecho de afrontar de manera simultánea el rol de estudiante y padres de familia, y el poco tiempo de que se dispone para el descanso o realizar actividades recreativas, son los principales aspectos detonantes del estrés. El manifestar este tipo de estrés laboral de una manera recurrente o casi permanente los vuelve más propensos a desarrollar el Síndrome de Burnout.

En la década de los ochenta, la psicóloga Cristina Maslach, en colaboración con S.E. Jakson, conceptualizaron al Síndrome de Burnout como una respuesta al estrés laboral crónico que se caracteriza porque: a) la persona desarrolla una idea de fracaso profesional, b) tiene la sensación de encontrarse emocionalmente agotado, y c) despliega actitudes negativas hacia las personas con las que trabaja ${ }^{1}$. Estos síntomas han dado lugar a un constructo tridimensional que integra:

- Agotamiento emocional: esta dimensión se refiere a la disminución y pérdida de recursos emocionales. Este es un componente fundamental del Burnout que puede tener manifestaciones físicas y psíquicas.

- Despersonalización: esta dimensión consiste en el desarrollo de actitudes negativas, entre ellas insensibilidad y cinismo, hacia los receptores del servicio prestado. La despersonalización, en un 
nivel moderado, es una respuesta adaptativa de los profesionales, pero esta misma respuesta, en grado excesivo, evidenciaría sentimientos patológicos hacia los demás².

- Baja realización personal: esta dimensión implica la tendencia a evaluar el propio trabajo de forma negativa; se tiene la sensación de insuficiencia profesional y una baja autoestima personal.

Este Síndrome ha sido estudiado en diferentes tipos de profesionistas, como es el caso de los policías ${ }^{3}$, teleoperadores ${ }^{4}$, docentes ${ }^{5}$, sacerdotes ${ }^{6}$, oficiales de prisiones ${ }^{7}$, entrenadores deportivos ${ }^{8}$, y médicos ${ }^{9}$, entre otros. Entre los profesionistas más estudiados, con relación a este síndrome, se encuentran los que laboran en el ámbito de la salud como sería el caso del personal de enfermería ${ }^{10}$, residentes de ortopedia $^{11}$, psicólogos ${ }^{12}$ y médicos ${ }^{13}$. En el caso del personal de enfermería, que es el de interés para la presente investigación, el síndrome de Burnout ha sido estudiado con relación a diferentes variables como las sociodemográficas, laborales y organizacionales.

De las variables sociodemográficas una que se estudia de manera frecuente es el sexo, con el fin de determinar cuál es más propenso a padecerlo; en algunas investigaciones son las mujeres quienes en mayor medida lo presentan ${ }^{14,15}$, mientras en otras son los hombres ${ }^{16,17}$. Por otra parte, con relación a la edad, se ha identificado que el tener entre 31 a 35 años $^{14}$ o una edad avanzada ${ }^{15}$ genera la aparición de este síndrome.

Con relación a las variables organizacionales los estudios reportan que la interacción conflictiva, la monotonía de la tarea y la falta de cohesión ${ }^{18,19}$, así como el conflicto de rol y la escasa o nula motivación laboral ${ }^{20,21}$ son detonantes del síndrome de Burnout.

En el caso de las variables laborales, diversos estudios indican que laborar en los turnos nocturno y especial ${ }^{10} \mathrm{o}$ fijo ${ }^{15}$, el personal de enfermería es más propenso a padecer el síndrome de Burnout; algo similar sucede con las áreas o servicios de administración/oncología/camillero/esterilización ${ }^{16}$, terapia intensiva ${ }^{14}$, urgencias ${ }^{20}$, mientras que otros estudios reportan mayor presencia de Burnout en servicios de emergencia y cuidados intensivos ${ }^{19}$.

La situación laboral es otra variable estudiada en este ámbito, a este respecto se considera que el personal de enfermería que tiene una vinculación temporal o de supernumerario (de contrato temporal) con la institución, así como más de un contrato suelen presentar con mayor frecuencia este síndrome ${ }^{16}$. Otros autores reconocen el papel que puede jugar la antigüedad en el trabajo ${ }^{14,15,22}$, ya que el personal de enfermería suele presentar un mayor nivel de Burnout al tener una antigüedad en la institución entre 11 y 15 años, o una antigüedad de 6 a 10 años en el mismo servicio o área de trabajo ${ }^{14}$. Por último, aspectos como mayor sueldo, satisfacción laboral alta y apoyo de la organización sirven para que este síndrome se presente en menor nivelel 23.

Estas investigaciones realizadas con personal de enfermería, muestran contradicciones importantes respecto al papel de las variables turno, sexo y tipo de servicio, entre otras, con relación al síndrome de Burnout; así mismo se puede observar que las condiciones laborales no han sido suficientemente exploradas, por lo tanto hacen falta mayores estudios al respecto.

Tradicionalmente, la mayoría de los estudios sobre el estrés, y variables asociadas como es el caso del síndrome de Burnout, se han centrado en el papel de ciertos rasgos de personalidad como variables predictoras, tal es el caso del neuroticismo y la indefensión aprendida, entre otras; sin embargo, existe otra perspectiva denominada contextualista ${ }^{24}$ en la que se asigna un papel más protagónico a la naturaleza de la situación, esto es, a las características contextuales. Bajo esta perspectiva, y ante la ausencia de datos concluyentes, en esta investigación el objetivo es identificar los rasgos del síndrome de Burnout y determinar si las variables: turno de trabajo, situación laboral, el realizar horas de trabajo extras y servicio en el que se encuentra actualmente, establecen diferencias en el nivel con que se presentan las tres dimensiones del síndrome de Burnout, en los profesionales de enfermería del Hospital Materno Infantil de la Ciudad de Durango. 


\section{Método}

El presente estudio, es de tipo transversal, correlacional (a través de un diseño de diferencia de grupos sin atribución causal) y no experimental, se contó con la participación de 204 enfermeras/os del Hospital Materno Infantil de la ciudad de Durango, en México. En total laboran en este hospital 288 enfermeras/os en los tres turnos y se intentó encuestar a la totalidad pero solamente 204 tuvieron disposición y/o disponibilidad para responder el cuestionario, por lo que no se obtuvo un $100 \%$ de cuestionarios respondidos. El principal problema para la recolección de información fue la disponibilidad de tiempo, ya que su aplicación fue en un momento de gran demanda laboral cuando los profesionales en enfermería estaban casi el 100\% de su jornada en contacto directo con el paciente, en el cual monitorizaban los signos vitales y los cambios en el patrón del estado de salud.

En la presente investigación se utilizó el instrumento Maslach Burnout Inventory-General Survey $\left(\right.$ MBI-GS) ${ }^{25}$ (Tabla 1), el cual fue respondido de manera autoadministrada y voluntaria en las mismas instalaciones del hospital en los diferentes turnos en que se labora.

Este Inventario comprende tres dimensiones:

- El cansancio emocional (CE): se manifiesta en sentimientos de estar emocionalmente agotado y exhausto por el propio trabajo con personas (ítems: 1,2,3,4 y 6); esta dimensión presenta un alfa de Cronbach de 0.72 .

- La despersonalización (DP): representa la falta de sentimiento y poca respuesta hacia los que reciben el servicio social que da el profesional (ítems: 8,9,13,14 y 15); dicha dimensión tiene un alfa de Cronbach de 0.64 .

- La baja realización personal (RP): consiste en la ausencia de sentimiento de competencia y éxito en la labor profesional con personas (ítems: 5,7,10,11,12 y 16); esta dimensión presenta un alfa de Cronbach de 0.85 .

La prueba consta de 16 ítems con opciones de respuesta en una escala tipo Likert de cuatro valores: nunca (1), casi nunca(2), algunas veces (3) y casi siempre(4); la respuesta indica la frecuencia con que se presenta cierta conducta o sentimientos en el encuestado.

En el cuestionario se agregaron tres ítems para la caracterización de la población (sexo, edad y antigüedad) (Tabla 2) y cuatro ítems para el análisis correlacional (turno de trabajo, situación laboral, el realizar horas laborales extras y servicio en el que se encuentra actualmente) (Tabla 3).

El presente trabajo se realizó en el Hospital Materno Infantil de la Ciudad de Durango, previa solicitud por escrito al citado Hospital; se acude a los diferentes servicios con los que cuenta esta institución para contactar al personal que participó en este estudio. Posteriormente se aplica el cuestionario a la muestra seleccionada explicándoles el objetivo de dicha investigación.

El proyecto contó con la autorización del Comité de Posgrado de la Universidad Pedagógica de Durango y se siguieron los protocolos del centro de trabajo donde se aplicó la encuesta, relativos a la publicación de datos del personal. En ninguna de las dos instituciones existe la figura de Comité de Ética, por lo que estos aspectos de la investigación, solamente los supervisa de manera general el Comité de Posgrado de la Universidad Pedagógica de Durango.

$\mathrm{Al}$ ser un estudio ex post facto, a través de la aplicación de encuesta, no existieron riesgos para el personal de enfermería involucrado. En el cuestionario a llenar no se les solicitó ningún dato que pudiera comprometer su anonimato y se les hizo saber que solo se manejarían resultados globales, como se puede observar en el presente escrito; también se les indicó, en la misma presentación del cuestionario, que la respuesta era voluntaria y estaban en su derecho de responderlo, o de no hacerlo, por lo que a través de esta información se obtuvo el consentimiento informado de manera indirecta. Los cuestionarios aplicados están en posesión del primer autor y se conservarán en resguardo el tiempo adecuado para ello. 
Se elaboró una base de datos mediante el paquete estadístico SPSS, Versión 21. Se realiza análisis descriptivo, con el uso de la media aritmética, como medida de tendencia central, y la desviación típica estándar, como medida de dispersión. Para el análisis correlacional se utilizó la t de Student (variables: situación laboral y horas extras) y ANOVA (variables: turno y servicio). En todos los caso la regla de decisión fue $\mathrm{p}<.05$.

\section{Resultados}

La distribución del personal de enfermería, según sus variables sociodemográficas, fue la siguiente: el $11.3 \%$ corresponden al sexo masculino y el restante $88.7 \%$ al sexo femenino. La edad mínima de los encuestados fue de 22 años y la máxima de 64, el promedio fue de 37 años. La antigüedad mínima en el servicio es de un año y la máxima de 43 , con promedio de 13 años de antigüedad.

Con relación a las variables situacionales de índole laboral su distribución fue la siguiente:

- De lunes a viernes, el 34.3\% labora en el turno matutino, el 13.2\% en el vespertino, el 29.9\% en el turno nocturno; para el fin de semana y días festivos el 15.7\% trabaja en la jornada de día y el $6.9 \%$ en la jornada nocturna.

- El 43.6\% tiene base (tipo de plaza que otorga todos los derechos y beneficios laborales a los trabajadores sindicalizados) y el $56.4 \%$ trabaja por contrato (plaza que carece de derechos y beneficios laborales).

- El $28.8 \%$ trabaja horas extras y el $71.2 \%$ no lo hace.

- El 8.8\% presta su servicio en Urgencias Pediátricas, el 7.8\% en Anti alacrán (área destinada a suministrar el antídoto contra los piquetes de alacrán), el 11.8\% en Toco Cirugía, el 6.4\% en Quirófano, el 5.9\% en CEyE (Central de Equipos y Esterilización), el 4.4\% en Unidad de Terapia Intensiva de Adultos (UTIA), el 9.3\%, en el servicio de Pediatría, el 7.8\% en Unidad de Terapia Intensiva Pediátrica (UTIP), el 18.6\% en Neonatos, el 9.3\% en Ginecología, el 2.5\% en Habitación Conjunta, el 1\% en Administración (Supervisión de enfermería), el 3.9\% en Inhaloterapia y con un $0.5 \%$ en los servicios de Central de Mezclas, Clínica Catéter, Microbiología, Clínica heridas y estomas.

Los resultados obtenidos del análisis descriptivo del MBI-GS se presentan en la Tabla 1, las medias altas se ubican en el ítem 2 Me siento acabado al final de la jornada diaria el que arroja una media de 2.48 (62\%), en seguida, con un 2.36 (59\%) se encuentra el ítem 13 Solo quiero hacer mi trabajo y que no me molesten; mientras que las medias más bajas se ubican en el ítem 10 En mi opinión, soy muy bueno haciendo mi trabajo con una media de 1.42 (35\%), el ítem 11 Me siento realizado cuando llevo a cabo algo en mi trabajo con un 1.49 (37\%) y finalmente en el ítem 12 He realizado muchas cosas que valen la pena en mi trabajo con una media de 1.58 (39\%).

En lo que respecta a cada una de las dimensiones constitutivas del síndrome de Burnout, los resultados indican que existe un mayor nivel de presencia de la dimensión cansancio emocional con una media de 2.27 (56\%), seguida por la dimensión despersonalización con una media de 1.84 (46\%), y al final se presenta la dimensión falta de realización personal con una media de 1.61 (40\%).

Estos resultados descriptivos, transformados a porcentaje y ubicados entre paréntesis enseguida de la media informada para cada caso (como se mostró en los dos párrafos previos), se pueden interpretar con el baremo recomendado por Barraza, Malo y Rodríguez ${ }^{26}$ : de 0 a $25 \%$ no hay burnout, de $26 \%$ a $50 \%$ se presenta con un nivel leve, de 51 a $75 \%$ se presenta con un nivel moderado y de $76 \%$ a $100 \%$ con un nivel fuerte o profundo; por lo que la dimensión cansancio emocional y el ítem Me siento acabado al final de la jornada diaria son los únicos elementos que se presentan con un nivel moderado, mientras que el resto de las dimensiones e ítems mencionados se presentan con un nivel leve.

Los resultados del análisis de diferencia de grupos, sin atribución causal, entre los rasgos del síndrome de Burnout y las cuatro variables situacionales, de índole laboral, se muestran en la Tabla 2. A partir de estos resultados se puede plantear el siguiente perfil: 
Tabla 1. Análisis descriptivo de ítems del Maslach Burnout Inventory-General Survey

\begin{tabular}{lcc}
\hline Ítem & Media & Desv. típ. \\
\hline Me siento emocionalmente agotado/a por mi trabajo & 2.34 & .952 \\
Me siento acabado al final de la jornada diaria & $\mathbf{2 . 4 8}$ & $\mathbf{. 8 9 0}$ \\
Me siento fatigado al levantarme por la mañana y tener & 2.10 & .928 \\
que enfrentarme otro día al trabajo & & 1.039 \\
Trabajar todo el día realmente es estresante para mí & 2.12 & 1.044 \\
Soy capaz de resolver eficazmente los problemas que surgen en mi trabajo & 1.70 & .978 \\
Me siento desgastado por mi trabajo & 2.32 & 1.052 \\
Siento que estoy haciendo una contribución eficaz a la actividad de mi organización & 1.81 & .958 \\
Desde que comencé en este empleo, he ido perdiendo el interés en mi trabajo & 1.62 & 1.132 \\
He ido perdiendo el entusiasmo en mi trabajo & 1.90 & .788 \\
En mi opinión, soy muy bueno haciendo mi trabajo & 1.42 & .885 \\
Me siento realizado cuando llevo a cabo algo en mi trabajo & 1.49 & 1.58 \\
He realizado muchas cosas que valen la pena en mi trabajo & .914 \\
Solo quiero hacer mi trabajo y que no me molesten & $\mathbf{2 . 3 6}$ & $\mathbf{1 . 0 9 4}$ \\
Me he vuelto más cínico acerca de si mi trabajo vale para algo & 1.71 & 1.025 \\
Dudo sobre el valor de mi trabajo & 1.64 & .995 \\
En mi trabajo estoy seguro de que soy eficaz haciendo las cosas & 1.69 & 1.059 \\
\hline
\end{tabular}

\section{Tabla 3. Nivel de significación del análisis de diferencia de grupos entre} las dimensiones del Burnout y las cuatro variables laborales

\begin{tabular}{llccc}
\hline Ítem & Turno & \multicolumn{2}{c}{$\begin{array}{l}\text { Situación } \\
\text { laboral }\end{array}$} & Horas extras \\
\hline Cansancio emocional & .768 & .008 & .440 & .307 \\
Despersonalización & .000 & .030 & .037 & .933 \\
Falta de realización personal & $\mathbf{. 0 0 0}$ & $\mathbf{. 0 1 8}$ & .060 \\
\hline
\end{tabular}

- El personal de enfermería que trabaja la jornada nocturna presenta con mayor nivel los siguientes rasgos del síndrome de Burnout: Me siento fatigado al levantarme por la mañana y tener que enfrentarme otro día al trabajo, Soy capaz de resolver eficazmente los problemas que surgen en mi trabajo, Siento que estoy haciendo una contribución eficaz a la actividad de mi organización, Desde que comencé en este empleo, he ido perdiendo el interés en mi trabajo, He ido perdiendo el entusiasmo en mi trabajo, En mi opinión, soy muy bueno haciendo mi trabajo, Me siento realizado cuando llevo a cabo algo en mi trabajo, He realizado muchas cosas que valen la pena en mi trabajo y En mi trabajo estoy seguro de que soy eficaz haciendo las cosas. En todos estos casos el personal de enfermería que trabaja en el turno matutino es el que menos presenta este tipo de rasgos. Los rasgos enunciados corresponden de manera indistinta a las tres dimensiones del síndrome de Burnout.

- El personal de enfermería que es de base presenta con mayor nivel los siguientes rasgos positivos: En mi trabajo estoy seguro de que soy eficaz haciendo las cosas, y Siento que estoy haciendo una contribución eficaz a la actividad de mi organización. Como se puede observar ambos ítems corresponden a la dimensión de realización profesional, por lo que se puede afirmar que el personal de base tiene, en estos ítems, un mayor nivel de realización profesional. 
- El personal de enfermería que su régimen laboral es de contrato presenta con mayor nivel los siguientes rasgos del síndrome de Burnout: Me siento acabado al final de la jornada diaria, Me he vuelto más cínico acerca de si mi trabajo vale para algo y Dudo sobre el valor de mi trabajo. Estos ítems pertenecen a las dos dimensiones negativas del Burnout: despersonalización y cansancio emocional.

El servicio donde se labora influye en tres conductas del síndrome de Burnout, aunque, no se pudo realizar la prueba post hoc y solamente se realizó una tabulación cruzada, a partir de ella se puede concluir que:

- El personal de enfermería que labora en Urgencias, Toco Cirugía (área donde se proporciona atención médica a pacientes gineco-obstetras, ya sea cirugía, parto o puerperio) y Terapia Pediátrica, son los que reportan que se sienten acabados al final de la jornada diaria.

- El personal de enfermería que labora en Toco Cirugía y Pediatría se sienten desgastados por el trabajo.

- El personal de enfermería que labora en Urgencias, Toco Cirugía y Quirófano, son los que menos consideran que han realizado muchas cosas que valen la pena en su trabajo

Por su parte, el análisis de diferencia de grupos, sin atribución causal, entre las dimensiones constitutivas del síndrome de Burnout y las cuatro variables laborales, se presentan en la Tabla 3. Como se muestra: a) el cansancio emocional es influido exclusivamente por la situación laboral, y es el personal de enfermería de contrato los que presentan mayor cansancio emocional; b) la despersonalización tiene influencia de las variables turno, situación laboral y horas extras, se advierte que el personal de contrato, que realiza horas extras y de jornada nocturna son los que la presentan con mayor frecuencia; y c) la falta de realización personal es influida por las variables turno y situación laboral, esto puede apreciarse en mayor medida en el personal de base y de jornada nocturna.

\section{Discusión}

Los profesionistas del área de la salud son de los más propensos a presentar estrés; entre ellos cabe destacar al personal de enfermería. A esta situación, inherente a su profesión, habría que agregar que en el contexto de la actual crisis económica, y de las reformas al sector salud, el personal de enfermería advierte un fuerte deterioro de sus condiciones laborales lo cual incrementa su nivel de estrés.

Cuando ese estrés se vuelve permanente desencadena una serie de reacciones y problemáticas, entre las que cabe mencionar el síndrome de Burnout. En la presente investigación se pudo constatar que existen ciertos rasgos de este síndrome que se presentan con mayor frecuencia como son "Me siento acabado al final de la jornada diaria", que corresponde a la dimensión cansancio emocional, y "Solo quiero hacer mi trabajo y que no me molesten", que corresponden a la dimensión de despersonalización. Ambas dimensiones constituyen el núcleo clave y le dan su carácter negativo a este síndrome.

Entre los rasgos que se presentan con menos frecuencia se ubican "En mi opinión, soy muy bueno haciendo mi trabajo" y "Me siento realizado cuando llevo a cabo algo en mi trabajo"; ambos rasgos pertenecen a la dimensión de realización personal por lo que su baja presencia implicaría la falta de realización personal. Este resultado coincide con el análisis realizado por dimensiones donde el cansancio emocional y la despersonalización tienen una mayor prevalencia que la falta de realización personal.

El hecho de que exista una mayor presencia de las dimensiones cansancio emocional y despersonalización y una menor presencia de la dimensión de realización personal coincide con estudios previos $^{25}$.

Si conjuntamos una alta presencia de los rasgos negativos del Burnout y una baja presencia de los rasgos positivos indagados en este síndrome, se puede afirmar una presencia clara de este síndrome en el personal encuestado, lo cual coincide con investigaciones precedentes ${ }^{14,15,21,22}$. Este nivel de 


\begin{tabular}{|c|c|c|c|c|}
\hline Ítem & Turno & $\begin{array}{l}\text { Situación } \\
\text { laboral }\end{array}$ & Horas extras & Servicio \\
\hline Me siento emocionalmente agotado/a por mi trabajo & .240 & .093 & .327 & .165 \\
\hline Me siento acabado al final de la jornada diaria & .664 & .000 & .334 & .001 \\
\hline $\begin{array}{l}\text { Me siento fatigado al levantarme por la mañana } \\
\text { y tener que enfrentarme otro día al trabajo }\end{array}$ & .035 & .381 & .776 & .444 \\
\hline Trabajar todo el día realmente es estresante para mí & .338 & .115 & .648 & .175 \\
\hline $\begin{array}{l}\text { Soy capaz de resolver eficazmente los } \\
\text { problemas que surgen en mi trabajo }\end{array}$ & .000 & .018 & .918 & .091 \\
\hline Me siento desgastado por mi trabajo & .730 & .211 & .955 & .045 \\
\hline $\begin{array}{l}\text { Siento que estoy haciendo una contribución } \\
\text { eficaz a la actividad de mi organización }\end{array}$ & .000 & .028 & .414 & .100 \\
\hline $\begin{array}{l}\text { Desde que comencé en este empleo, he ido } \\
\text { perdiendo el interés en mi trabajo }\end{array}$ & .000 & .241 & .305 & .050 \\
\hline He ido perdiendo el entusiasmo en mi trabajo & .000 & .643 & .125 & .447 \\
\hline En mi opinión, soy muy bueno haciendo mi trabajo & .000 & .442 & .759 & .161 \\
\hline Me siento realizado cuando llevo a cabo algo en mi trabajo & .001 & .195 & .662 & .115 \\
\hline He realizado muchas cosas que valen la pena en mi trabajo & .000 & .061 & .744 & .008 \\
\hline Solo quiero hacer mi trabajo y que no me molesten & .938 & .204 & .693 & .365 \\
\hline Me he vuelto más cínico acerca de si mi trabajo vale para algo & .060 & .037 & .167 & .767 \\
\hline Dudo sobre el valor de mi trabajo & .003 & .002 & .050 & .287 \\
\hline En mi trabajo estoy seguro de que soy eficaz haciendo las cosas & .000 & .045 & .353 & .075 \\
\hline
\end{tabular}

presencia del síndrome de Burnout es preocupante por los efectos que causa al desempeño profesional de los enfermeras/os, ya que como afirma la Organización Mundial de Salud: los trabajadores que muestran estos síntomas tendrán un impacto negativo en la productividad y la calidad del trabajo, por lo que afectarán directamente a la empresa ${ }^{27}$.

En el caso de las cuatro variables laborales indagadas, con relación al síndrome de Burnout, fue sin duda el turno y la situación laboral las que ejercen una mayor influencia en la generación de este síndrome. Esto coincide con otras investigaciones previas ${ }^{10,15,16}$. En lo que respecta a la situación laboral, el presente estudio reporta que el personal de base tiene varios rasgos que indican un mayor nivel de falta de realización personal, en comparación con los que están por contrato, y que los que están bajo contrato presentan mayor prevalencia en las dimensiones despersonalización y cansancio emocional; este resultado coincide parcialmente con los estudios que afirman que el personal de enfermería que no tiene base laboral presenta con mayor frecuencia este síndrome ${ }^{16}$.

Por otra parte, se puede decir que los servicios o áreas donde labora el personal de enfermería tienen una influencia en la aparición del síndrome de Burnout, aunque en la presente investigación solo se presente en tres rasgos, por lo que se coincide, en parte con resultados previos con relación al área de urgencias ${ }^{20}$ y se difiere con los reportados en otras investigaciones ${ }^{19}$. Sin lugar a dudas la presencia de este síndrome difiere, de forma significativa según el servicio o área en donde labora ${ }^{20}$, aunque los resultados se muestren diversos y contradictorios.

Cabe resaltar que la variable trabajar horas extras no había sido indagada con anterioridad y los resultados de la presente investigación indican que no se relaciona con ningún rasgo del síndrome de 
Burnout, pero si con la dimensión de despersonalización, por lo que se puede afirmar que el trabajar horas extras hace que el personal de enfermería presente mayor despersonalización al tratar a los pacientes. De igual forma, aparecen en los resultados las áreas de Toco Cirugía, Pediatría y Quirófano que presentan con mayor frecuencia ciertos rasgos de este síndrome; estos servicios no habían sido mencionados en estudios previos.

Estos resultados, junto a otros ${ }^{10,14-16,18-22}$, adquieren relevancia si lo que se busca es un entorno de trabajo saludable, entendido éste como aquel en el que los trabajadores y jefes tienden a colaborar en prácticas de mejora continua con el objetivo explícito de promover y proteger la salud, seguridad y bienestar de los propios trabajadores ${ }^{27}$, sobre todo en lo concerniente al medio psicosocial del trabajo que incluye la organización del mismo, las relaciones interpersonales y la cultura del ambiente de trabajo. En este caso, las condiciones laborales u organizacionales pueden ser generadoras del síndrome de Burnout y afectar la salud y bienestar mental de los trabajadores, lo cual tiende a tener un impacto negativo en el desempeño profesional.

\section{Conclusiones}

A pesar de que, aparentemente, el estudio de variables antecedentes de este síndrome ya ha sido suficientemente abordado, y aparecen en las revisiones teóricas o estados del arte elaborados al respecto $^{28}$, es necesario considerarlas poco exploradas por sus resultados no concluyentes. En el caso de la presente investigación se puede concluir que las variables laborales estudiadas (turno de trabajo, situación laboral, el realizar horas laborales extras y servicio en el que se encuentra actualmente), pueden marcar una diferencia significativa en el nivel con que se presentan algunos rasgos o dimensiones del Burnout; sin embargo, estos resultados siguen marcando el carácter complejo y multifacético con que aparecen las variables antecedentes de dicho síndrome, sean estas sociodemográficas, laborales u organizacionales por lo que se considera necesario continuar con mayores estudios al respecto, a fin de que la acumulación de evidencia empírica ayude a determinar cuáles son verdaderamente las variables antecedentes del síndrome.

Una explicación tentativa a la dispersión y contradicciones propias en este campo obedece a la presencia de diferentes niveles y formas de análisis cuando se aborda su estudio: algunos lo analizan como variable individual, mientras otros lo hacen como variable colectiva. Unos analizan los rasgos del Burnout, otros sus dimensiones y unos más lo intentan abordar como variable general. Esto sin lugar a dudas es algo a tener en cuenta en futuras investigaciones. En el caso de la presente investigación se decidió no abordar el nivel global del síndrome de Burnout ante los fuertes cuestionamientos que hay sobre si realmente puede ser considerada una variable general o tres variables integradas en un síndrome; por lo que el análisis se realizó a partir de sus tres dimensiones, al igual que estudios anteriores.

A manera de cierre cabe destacar como principal limitación de este estudio, el hecho de no haber podido obtener el $100 \%$ de cuestionarios respondidos del personal de enfermería en los diferentes servicios del hospital donde se aplicó; además, el cuestionamiento al bajo de nivel de confiabilidad reportado en algunas de las dimensiones del instrumento utilizado para indagar este síndrome. Por el contrario, su principal fortaleza es la de continuar la línea de investigación abierta por estudios preliminares sobre el papel de las variables laborales en la generación de este síndrome, sobre todo el estudio de algunas variables que no habían sido estudiadas previamente. Con esto se busca contribuir al acervo empírico del campo lo que permitirá continuar incentivando la indagación al respecto.

\section{Responsabilidades Éticas}

Protección de personas y animales. Los autores declaran que para esta investigación no se han realizado experimentos en seres humanos ni en animales.

Confidencialidad de los datos. Los autores declaran que se han seguido los protocolos de su centro de trabajo sobre la publicación de datos de pacientes. 
Derecho a la privacidad y consentimiento informado. Los autores declaran que en este artículo no aparecen datos de pacientes.

Financiamiento. No se ha contado con ningún tipo de financiamiento.

Conflicto de intereses. No hay ningún conflicto de intereses.

\section{Referencias}

1. Maslach C, Jackson SE. Maslach Burnout Inventory. $2^{\mathrm{a}}$ ed. California: Consulting Psychologist Press; 1986.

2. Oliver C. Análisis de la problemática de estrés en el profesorado de enseñanza media: el burnout como síndrome específico. [tesis de doctorado] Madrid: Facultad de Psicología de la Universidad Autónoma de Madrid; 1993.

3. Escudero J, Delfín LA, Arano R. Estudio de la relación entre el síndrome de quemado por el trabajo (Burnout) y la antigüedad laboral, en el personal policiaco operador de atención telefónica ciudadana de un estado del sureste de México, período 2014-2015. Ciencia Administrativa. 2015; 2: 20-8.

4. Muñoz ME. Propiedades psicométricas del inventario de burnout de Maslach en teleoperadores de dos empresas de Trujillo. [tesis de licenciatura]. Lima: Universidad César Vallejo; 2016.

5. Torres P, Cobo J. Estrategias de gestión de la inteligencia emocional para la prevención del Síndrome de Burnout en docentes de aula. Educ@ción en Contexto. 2016; 2(especial): 281-95.

6. Manzanares E, Merino C, Fernández M. Estructura interna del Maslach Burnout Inventory (MBI) en una muestra de sacerdotes y religiosas católicas peruanos. Salud soc. 2016; 7(2): 198-211. http://dx.doi.org/10.22199/S07187475.2016.0002.00005

7. Álvarez PL, Chacón F, Sánchez E, et al. Burnout en funcionarios/as de prisiones en Chile. Revista Iberoamericana de Psicología: Ciencia Tecnología. 2016; 9(1): 63-72.

8. Pulido JJ, Sánchez-Oliva D, Leo FM, et al. Frustración de las necesidades psicológicas, motivación y burnout en entrenadores: incidencia de la formación. Rev. psicol. deport. 2017; 26(1): 27-36.

9. Alvarado, L. Desgaste profesional en médicos del Hospital Regional “Teodoro Maldonado Carbo” del Instituto Ecuatoriano de Seguridad Social Revista cubana de salud y trabajo. 2016; 17(2): 261-8.

10. Rodríguez-López AI, Méndez-Durán A. Síndrome de burnout en profesionales de enfermería de áreas críticas en un hospital de tercer nivel. Gac Med Bilbao. 2016;113(3): 99-104.

11. Arboleda O. Síndrome de Burnout en residentes de ortopedia y traumatología en Colombia. [tesis de posgrado]. Bogotá: Universidad Nacional de Colombia; 2017.

12. Rodríguez SYS, Carlotto MS. Predictors of Burnout Syndrome in psychologists. Estud. psicol. 2017; 34(1): 141-50. http://dx.doi.org/10.1590/1982-02752017000100014

13. Tironi MOS, Teles JMM, Barros DS, et al. Prevalência de síndrome de burnout em médicos intensivistas de cinco capitais brasileiras. Rev Bras Ter Intensiva. 2016; 28(3): 270-7. http://dx.doi.org/10.5935/0103-507X.20160053

14. Juárez MG, Haros YH, Avalos JG, et al. Factores de riesgo relacionados con el Síndrome de Burnout en el personal de enfermería de un Hospital de Ciudad Juárez, Chihuahua, México. CULCyT. 2015; $57(2): 289-96$.

15. Valdivieso JF, Amador FJ, Hernández L, et al. Síndrome de Burnout en enfermeros que laboran en las unidades de cuidados intensivos, intermedios y coronarios del Hospital ‘Joaquín Albarrán’. Revista cubana de salud y trabajo. 2016;17(4): 3-11.

16. Grisales H, Muñoz Y, Osorio D, et al. Síndrome de Burnout en el personal de enfermería de un hospital de referencia Ibagué, Colombia, 2014. Enfermería glob. 2016; 41: 244-57. 
17. Martínez-López C, López-Solache G. Características del síndrome de Burnout en un grupo de enfermeras mexicanas. Arch. Med. Fam. 2005; 7(1): 6-9.

18. Barraza A, Carrasco R, Arreola MG. El síndrome de desgaste profesional en las enfermeras del Centro de salud No. 2 de la ciudad de Durango. Revista PsicologiaCientifica.com. 2008; 10 (25).

19. Hernández, S.M.R. Síndrome de burnout en enfermeras que laboran en emergencia y unidad de cuidados intensivos del Hospital Regional Docente las Mercedes Chiclayo, enero 2015. [tesis de grado]. Pimentel: Universidad Señor de Sipán; 2015.

20. Muñoz AI, Velásquez MS. Síndrome de quemarse por el trabajo en profesionales de enfermería, Bogotá, Colombia. Rev. Fac. Nac. Salud Pública. 2016; 34(2): 202-11.

http://dx.doi.org/10.17533/udea.rfnsp.v34n2a09

21. Yslado R, Atoche R, Cermeño B, et al. Síndrome de burnout y factores sociodemográficosorganizativos en profesionales de salud. Hospitales del callejón de Conchucos, Ancash, Perú - 2012. Rev. investig. psicol. 2013; 16(1): 191-209. http://dx.doi.org/10.15381/rinvp.v16i1.3927

22. Arias WL, Muñoz A. Síndrome de burnout en personal de enfermería de Arequipa. RCSP. 2016; 42(4): 1-19.

23. Gómez-Urquiza JL, Monsalve-Reyes CS, San Luis-Costas C, et al. Factores de riesgo y niveles de burnout en enfermeras de atención primaria: una revisión sistemática. Aten. prim. 2017; 49(2): 77-85.

24. Aldwin CM, Revenson TA. Does coping help? A reexamination of the relation between coping and mental health. J Pers Soc Psychol. 1987; 53(2): 337-48.

25. Guevara LM, Ocampo N. Propiedades Psicométricas de Confiabilidad y Validez del Maslach Burnout Inventory - General Survey. Rev. Interam. Psicol. Ocup. 2014; 33(2): 128-42. http://dx.doi.org/10.21772/ripo.v33n2a04

26. Barraza A, Malo DA, y Rodríguez MR. Síndrome de burnout en estudiantes de dos facultades de medicina. Un estudio comparativo entre una institución colombiana y una mexicana. En: Barraza A, Malo DA, Investigaciones sobre salud mental. México: Red Durango de Investigadores Educativos A.C; 2012. 36-57.

27. World Health Organization. Entornos Laborales Saludables: Fundamentos y Modelo de la OMS. Contextualización, Prácticas y Literatura de Apoyo. Ginebra: OMS; 2010.

28. Gil-Monte PR, Peiró JM. Perspectivas teóricas y modelos interpretativos para el estudio del síndrome de quemarse por el trabajo. An. psicol. 1999; 15(2): 261-8. 